\section{Hugo von Hofmannsthals Kriegsziel-Notizen im Kontext deutscher und österreichischer Südosteuropa-Konzepte im Ersten Weltkrieg}

»Oesterreich als ein bloßes belastendes Hinterland [...] Oesterreich den Balkan und das oestliche Mittelmeer beherrschend « ${ }^{1}$

1.

Obwohl Hugo von Hofmannsthal nach rasch erlangter Rückversetzung aus seiner Truppenzuteilung in Pisino/Pazin (Istrien), wo er bereits am 31. Juli 1914 nach Intervention aus dem Büro des Ministerpräsidenten Graf Stürckh, eingefädelt über Josef Redlich und Leo Chlumecky, eine Beurlaubung erhielt, um nach Wien ins Kriegsfürsorgeamt überstellt und am 1. Oktober 1914 aufgrund einer »allgemeinen Nervenschwäche mittleren Grades mit Beteiligung des Herzens « als zum Truppendienst untauglich erklärt zu werden, ${ }^{2}$ entwickelte er seit Anfang September

1 Hofmannsthal: Aufzeichnungen, Nr. 1352, S. 655. Notizen vom 28.10.1915, anlässlich eines Treffens mit deutschen Pressevertretern in Brüssel.

2 Vgl. Lunzer: Hofmannsthals politische Tätigkeit, S. 28f. Symptomatisch dazu auch die Bemühungen Hofmannsthals, im
Ausgehend von den im Zuge der kritischen Werkausgabe erstmals vollständig edierten Notizen und Aufzeichnungen Hofmannsthals zum Ersten Weltkrieg befasst sich der Beitrag mit dem Stellenwert und der Art dieser Notizen und Reflexionen. In weiterer Folge werden diese mit den für die "Neue Freie Presse" verfassten Kriegsfeuilletons sowie mit den relevanten Briefwechseln (v.a. mit E. Bodenhausen, Harry Graf Kessler, J. Redlich) abgeglichen. Schließlich rekonstruiert der Beitrag die bei Hofmannstahl nachweisbaren Lektüren zeitgenössischer Kriegszielpublikationen und kontrastiert sie mit seinen eigenen, stärker kulturpolitisch ausgerichteten Vorträgen und Publikationsvorhaben zwischen 1915 und 1917. 
1914 eine frenetische publizistisch-propagandistische Aktivität. Diese wird gewöhnlich unter das Motto eines seiner frühen einschlägigen Texte, d.h. Die Bejahung Österreichs gestellt, und hat Karl Kraus anlässlich des Erscheinens der Österreichischen Bibliothek (1916) veranlasst, Hofmannsthal einerseits vorzuhalten, sich »in einer ziemlich versteckten Filiale des Krieges bequem gemacht « zu haben. Andererseits hat er ihm aufgrund der besonderen "Gnade des Schicksals oder wie die Protektion sonst heißen mag « die Berechtigung abgesprochen, in großen Reden »die Nation zu vertreten . $^{3}$

Bildet der Kriegsausbruch auf der Grundlage der neu edierten Aufzeichnungen zunächst eine auffällige Leerstelle, die im Kontrast zu recht unterschiedlichen, tendenziell eher dynamischen Korrespondenzen steht, so sucht Hofmannsthal über diesen `Glauben an Österreich〈, »eine complicierte Idee«, wie er anmerkt, eine Form von Stabilität zu finden. ${ }^{4}$ Begleitet ist diese Anstrengung von einer bemerkenswerten Parallelisierung zweier Wahrnehmungsmodi: einem stichwortartigen Schlachtenbulletin einerseits und dem bedauernden Feststellen, seit »Kriegsbeginn [...] noch nicht geträumt « zu haben andererseits. ${ }^{5}$ Zahlreiche Eintragungen vermitteln dabei den Eindruck eines Changierens zwischen Auslagerungen ins FiktionalLiterarische und bündig-prägnanten Exkursionen in eine den Krieg aus Distanz beobachtende, mitunter auch kommentierende Haltung. Sprachbildlich doppeldeutig kodiert erscheint dieser Glaube an die >complicierte Idee bereits im ersten seiner »Neue Freie Presse«-Essays, in Appell an die Oberen Stände, in Szene gesetzt: mit einer Faust-Zitatreminiszenz - »Das völlig Unfaßliche ist Ereignis geworden $\aleph^{6}$ - sowie unterlegt mit bedrohlichen Dunkelbildern, aber auch einer »leben und leben zu lassen«-Perspektive, gemünzt auf eben jene `höheren Stände`, denen ein Beitrag zu den sich einstellenden Engpässen in der Versorgung abgefordert wird.

Mag Hofmannsthal Anfang September 1914 das Unfassliche in seinen realen Dimensionen, d.h. in der brutalen Materialisierung der Augusteuphorie - die er am Tag seiner Einberufung auf einer Postkarte an Ottonie Gräfin Degenfeld noch verewigt hatte mit den Worten: »Glauben Sie mir [...] daß wir alle hier, bis zum letzten Holzknecht, in diese Sache

März 1915 über Arthur Schnitzler ein Gutachten über seine »wirklich absurden Nerven« zu erhalten. Vgl. Hofmannsthal/Schnitzler: Briefwechsel, S. 277.

3 Kraus: Gruß an Bahr und Hofmannsthal, S. 48 bzw. S. 50; danach in: ders.: Weltgericht; dazu: Scheichl: Karl Kraus' Weltgericht - Eine Bilanz, S. $289 \mathrm{f}$.

4 Hofmannsthal: Aufzeichnungen, Nr. 1308 (vermutl. Nov.-Dez. 1914), S. 628.

5 Ebd., Nr. 1304, 2.11.1914, S. 627.

6 Hofmannsthal: Appell an die höheren Stände, S. 101; vgl. den Schlusschor in Goethe: Faust II, V. $12105 f$. 
[= Krieg, Anm. d. Verf.] und in alles, was daraus werden möge, mit einer Entschlossenheit, ja mit einer Freude hineingehen, wie ich sie noch nie erlebt habe... $\ll^{7}$ - zunächst eher fremd erschienen sein, so fing es alsbald an, sich einzunisten und an Gewicht zu gewinnen. Vordergründig ging es dem Dichter vermutlich um Aufrechterhaltung von Normalität in Zeiten des »Ungeheuerlichen «, das »um uns sich vollzieht», das im blassen Blick der ersten Verwundeten ebenso aufblitzte wie in der von ihm wiederholt bekräftigten Formel vom >leben und leben lassen.$^{8}$

Eine explizite Vorstellung, inwiefern sowohl das Unfassliche als auch der Glaube an Österreich mit der Realität des Krieges und der ihn treibenden, unergründlich wirkenden Interessenslagen verrechnet werden könne, lässt sich aus den ersten Kriegsaufsätzen und den meisten Annotationen bis Ende 1914, im erstaunlichen Gegensatz zu sowohl euphorischen als auch pragmatischen Briefkorrespondenzen, jedoch kaum ablesen. So schließen die Eintragungen zum Jahr 1914 denn auch mit einem Briefentwurf an die Gräfin Zichy. Darin ist unüberhörbar ein larmoyanter Zwischenton auszumachen, wenn es heißt: »Österreich hungert nach politischem Geist. Es ist nicht imstande, sich zu seiner Idee aufzuschwingen [...] «. ${ }^{9} \mathrm{Zu}$ einer Idee, die - im Nachhinein gesehen - beinahe pazifistisch klingt, insofern sie mit Ungarn eine "wirkliche Verbindung mit Europa $\aleph^{10}$ ermöglichen könnte. Oder naivfahrlässig angesichts der Ende 1914 bereits vorliegenden Verlustmeldungen, für Hofmannsthal abstrakte, heroische Chiffren à la `unerhörte Zähigkeit` wie sie Front-Berichte in der »Neuen Freie Presse« vermittelten, die sich ihm trotz privilegierten Informationszugangs, trotz regelmäßiger Treffen mit Diplomaten (neben Leopold von Andrian auch Georg von Franckenstein) und hohen Militärs nur schwer zu einem realitätsnahen Bild fügen wollten. ${ }^{11}$

\section{2.}

Dass Krieg war, daran erinnerten Hofmannsthal die täglichen Erfolgsmeldungen der "Neuen Freien Presse« - auch wenn sie de facto vor allem Misserfolge verschleierten; sodann die sich selbst gestellten Kriegs-Propa-

7 Hofmannsthal/Degenfeld: Briefwechsel, S. 304, Postkarte vom 28.7.1914 (Wien durchfahrend).

8 Hofmannsthal: Appell an die höheren Stände, S. 98 bzw. 100.

9 Hofmannsthal: Aufzeichnungen, Nr.1309, S. 629.

10 Ebd.

11 Vgl. »Neue Freie Presse« (1.1.1915), S. 1: (Anonym): Die Eröffnung der Siegel. Im Anschluss an diesen Leitartikel wurde dort Hofmannsthals Beitrag Aufbauen, nicht einreißen (S. 3), begleitet von Max Nordaus Feuilleton Silvesternacht (S. 1-3) abgedruckt. 
ganda-Publikationsvorhaben, die in einer Notiz Ende April 1915 als >Politische Aufsätze , insgesamt zwölf, aufgelistet und mit Freunden (Hermann Bahr, Josef Redlich) z.T. einlässlich besprochen werden; und schließlich einzelne seiner Korrespondenzen. Zu diesen zählten insbesondere die mit Harry Graf Kessler seit August 1914 wieder in Schwung gekommene sowie jene mit Eberhard von Bodenhausen ab Oktober 1914. Bodenhausen gegenüber formulierte Hofmannsthal dabei sehr früh geradezu visionäre, vom Gegenüber allerdings nicht direkt kommentierte Einschätzungen über einen, so seine Wahrnehmung, kolonialen Status Österreichs in seinem Verhältnis zu Deutschland: »... wir sind ja nichts anderes als eine riesengroße, maßlos schwierige deutsche Colonie mitten in Europa«. Im selben Brief lieferte er ihm zugleich gewünschte Angaben über nachgefragte GranatenProduktionsziffern mit Verweisen auf einschlägige Firmen, die - auch das symptomatisch - dem deutschen Zweifel an der Ernsthaftigkeit der österreichischen militärischen (Aus)Rüstung entsprungen waren. ${ }^{12}$

Kessler gegenüber, der sich bereits Tage vor den offiziellen Kriegserklärungen vom kosmopolitischen Kunstmäzen zum glühenden deutschen Patrioten gewandelt hatte, gestand Hofmannsthal, der im Zuge der Einberufung nach Pisino seine Verbindungen genützt hat, um sein Kriegsengagement auf ein erträgliches Maß zu reduzieren, verschüchtert ein, unter dem »Fernsein von der Front « zu leiden. ${ }^{13}$ Als gelte es, die bald einsetzende publizistische Arbeit durch >authentisches` Fronterleben abzustützen, folgten diesem Eingeständnis 1915 mehrere Annäherungsfahrten an die Schlachtfelder im Westen wie im Osten, die ihn im Juni 1915 bis Krakau und im Oktober desselben Jahres nach Brüssel führten, einschließlich eines »Ausflug[s] an die See-front $« .{ }^{14}$ Sie gipfelten in einem mehrwöchigen Aufenthalt in Berlin, wo Hofmannsthal, ohne zuvor jemals in unmittelbare Berührung mit dem Geschehen in den vorderen Linien gekommen zu sein, seine Kontakte mit zahlreichen `Experten der Etappe, aber auch mit (militär)politischen Entscheidungsträgern vertiefen konnte.

Es werden wohl auch drängende Fragen Kesslers - z.B.: »Was wollt ihr mit Serbien machen? « $^{15}$ - sowie Kesslers wie auch Bodenhausens nicht immer freundliche Äußerungen über den aus ihrer Sicht wenig berechen-

12 Hofmannsthal/Bodenhausen: Briefe der Freundschaft. Brief vom 7.10.1914, S. 170 bzw. 4.11.1914, S. $181 f$.

13 Vgl. Hofmannsthal/Kessler: Briefwechsel 1898-1929, Brief Kesslers vom 7.11.1914, S. 385. Es ist Kessler, der in diesem Brief offensichtlich auf eine Klage Hofmannsthals repliziert. Vgl. dazu die kompakte Darstellung von Propst, Maximilian: Harry Graf Kessler.

15 Ebd., S. 387. 
baren Bundesgenossen mitverantwortlich dafür gewesen sein, dass Hofmannsthal zwischen ausgedehnten literarischen und historischen Lektüren (Goethe, Hebbel, Immermann, Mommsen, Burckhardt, aber auch Balzac und Whitman) seine kulturpolitischen Überlegungen und Annotationen verstärkt auf ein vermutetes deutsches Unverständnis über die in seiner Sicht doch kompliziertere innere wie äußere Gemengelage der Monarchie, etwa in ihrem Verhältnis zu den slawischen Nationen, ausrichtete. Zugleich dürfte diese Wahrnehmung Hofmannsthal motiviert haben, einen Blick auf damals prominente deutsche Kriegszielliteratur zu werfen, konkret auf mindestens drei Texte: auf Karl v. Winterstettens Berlin-Bagdad (1914) sowie auf Max Schelers Der Genius des Krieges und Paul Rohrbachs Russland und wir, beide Anfang 1915 erschienen. Dies traf sich insofern gut, als Hofmannsthal seit Ende 1914 Gelegenheit hatte, wiederholt mit seinem Freund Leopold von Andrian die $>$ Lage $\triangleleft$ zu besprechen. Andrian, zu jener Zeit als Diplomat des k.u.k. Ministeriums des Äußeren in Warschau stationiert, war unter anderem mit der heiklen Aufgabe betraut, die Interessen Wiens beim deutschen Generalgouvernement zu vertreten (z.B. die Aufteilung der wichtigen Kohlengebiete von Dabrowa betreffend, was er sehr zum Missfallen seiner deutschen Gegenüber offenbar mit schlüssigen Argumenten tat). Inwieweit dabei das bereits Ende August 1914 von Andrian erstellte Memorandum über österreichische Kriegsziele Russland gegenüber, d.h. im Wesentlichen die sogenannten >Polnische Frage $\triangleleft$, die auch Auswirkungen auf Balkan-Konzepte gehabt hätte, mit Hofmannsthal besprochen wurde, entzieht sich mangels Aufzeichnungen und Briefhinweise unserer Kenntnis. ${ }^{16}$ Allerdings hat Hofmannsthal bereits im Vorfeld der Krakau-Reise mit Andrian mehrmals Kontakt gehalten, um einerseits die entsprechenden Genehmigungen zu erhalten, andererseits die publizistische Verwertung zu besprechen. ${ }^{17}$ Zudem hat Hofmannsthal Anfang 1915 mit dem Politiker und Juristen Josef Redlich Aspekte seiner Publikationen zum Krieg erörtert, z.B. zur Frage des Umgangs der Nationen miteinander - interessant dabei z.B. die Versuche Masaryk zu gewinnen - und somit einen Austausch über die österreichische Idee auch im Sinn einer kulturpolitischen Kriegszielreflexion angestrebt. Diesem Austausch vermochte sich Redlich allerdings nicht im erhofften Ausmaß zu öffnen, nicht zuletzt wegen gegenläufiger Einschätzung der ins Auge gefassten vormärzlichen Österreich-Kritiken 
von Victor von Andrian-Werburg und Karl Moering sowie der polnischen und südslawischen Frage, die ihm als Schreckvisionen vor Augen standen. ${ }^{18}$

Es war wohl auch die Wiederbegegnung mit Eberhard von Bodenhausen in Brüssel, der aufgrund seiner Position in der Familie Krupp über hervorragende Beziehungen zu den militärischen und wirtschaftlichen Eliten des Deutschen Reiches verfügte und selbst als Verfasser von Memoranden auftrat, die Hofmannsthals Reserven der forschen deutschen Kriegszielrhetorik und der Praxis der deutschen Militärbehörden gegenüber, z.B. in Russisch-Polen - »unmögliches der preussischen «, bei denen er als »unbewusst gewordene Staatsraison « eine grundlegende »Höflichkeit« im Umgang mit den Nationen vermisste ${ }^{19}$-, zunächst bekräftigt haben dürften. Diese Reserven werden im erwähnten Bericht über die Militärverwaltung in Polen an mehreren Stellen eingearbeitet und zur Stützung der Argumentation herangezogen. So könne »bei aller Improvisation «, von Hofmannsthal als »Gabe des Lebens« hochstilisiert, das von den k.u.k. Truppen okkupierte Gebiet doch eine "geregelte[n] Verwaltung" aufweisen, die dem im römischen Recht verankerten Prinzip der "Fürsorge eines guten Hausvaters" nachempfunden sei. Darüber hinaus zeichne sie eine von deutscher Praxis deutlich abgesetzte national-kulturelle Sensibilität aus. Im Verbund mit der Kompetenz der Armee und ihrer Verwaltungsstellen sei sie nämlich in der Lage, auch die Sprache der in den okkupierten Territorien Lebenden zu sprechen, - eine »oesterr. Specialität $«{ }^{20}$ so in den vorbereitenden Notizen:

Ihre Sprache sprechen, das ist noch nicht alles, doch es ist viel; noch anderes kommt dazu, eine gewisse Generosität des Herzens und jenes kaum zu Definierende, das doch im Zusammenleben der Nationen so schwer wiegt: Takt. Mehr als den Deutschen ist es uns gegeben, mit Fremden zu hausen: als Nachbarn, als Herren, als zeitweilige Verweser, als Freunde, wie immer. Hier liegt eine Tradition von vielen Jahrhunderten vor, die uns ins Blut gegangen ist. Aus ihr ziehen wir in Polen große Vorteile. ${ }^{21}$

\section{3.}

Gestützt auf diese selbstbewusste öffentliche Positionierung und unter Ausblendung der weniger rühmlichen Seiten der k.u.k. Kriegsführung im

Vgl. Andrian: Korrespondenzen, Notizen, Essays, Berichte, S. 235f. Dazu auch die Aufzeichnung N. 1313 vom April 1915 über Politik, Verwaltung und Kultur (Gespräch mit Andrian), S. 634, bzw. Gespräch mit Redlich vom 30.6.1915 über >Unsere Nationen` (ebd., S. 638) sowie Brief Redlich vom 23.10.1915, in: Hofmannsthal/Redlich: Briefwechsel, S. $22 \mathrm{f}$.

19 Vgl. Hofmannsthal: Aufzeichnungen, Nr. 1320, datiert mit Juni 1915, S. 637.

20 Hofmannsthal: Unsere Militärverwaltung in Polen, S. 168 bzw. 789.

21 Ebd., S.173. 
Osten, ${ }^{22}$ nützte Hofmannsthal im Zuge seines Brüssel nachfolgenden Berlinbesuchs (Ende Oktober 1915) eigene hochrangige Kontakte, etwa zu Paul Thun-Hohenstein, Attaché an der k.k. Botschaft in Berlin, um in Kreisen der Diplomatie, z.B. beim Botschafter Gottfried zu Hohenlohe-Schillingfürst, seine Vorstellungen über Möglichkeiten der Beeinflussung der deutschen öffentlichen Meinung über Österreich vorzutragen. ${ }^{23}$ Dabei war es wiederum Bodenhausen, der Hofmannsthal weitere prominente Kontakte wie z.B. ein Treffen mit dem für die Kriegsfinanzierung wichtigen Staatssekretär Karl Helfferich, ermöglichte, und ihm leitende Mitglieder der Deutschen Gesellschaft (in den Aufzeichnungen als >Club < bezeichnet) wie Karl G. Vollmöller oder den Kolonialstaatssekretär Wilhelm Solf vorstellte. Damit fand sich Hofmannsthal schlagartig aus den bislang eher zurückhaltend-defensiv agierenden k.u.k.- Kontexten ins Zentrum expansiver deutscher Kriegsplanungen und flankierender Kriegspublizistik katapultiert. Deren »Geist des Schützengrabens ${ }^{24}$ infizierte auch Hofmannsthal kurzzeitig und zwar in zweierlei Hinsicht. Einerseits, um im Rahmen seiner >Unsere Nationen $<-$ Überlegungen die Differenzen zu den Deutschen nochmals und zum Teil sehr dezidiert zu markieren - allen voran ihnen einen »Mangel an Tact» (»Die Deutschen müssen selbst um Maass ringen«) zu attestieren, - nicht ohne zugleich deren Energien mit heimlicher Bewunderung anzuerkennen: "wir müssen preussischer werden und zugleich österreichischer «. ${ }^{25}$

Weitere Notizen legen nahe, dass die Reflexionen wohl auch darauf zielten, Bausteine für eine allerdings unveröffentlicht gebliebene >vertrauliche Skizze über die Möglichkeit der Beeinflussung der öffentlichen Meinung in Deutschland bereitzustellen, und zwar gerade zu einer Zeit, als Bestrebungen im Gang waren, das Flaggschiff der österreichischen Zeitungslandschaft, die »Neue Freie Presse«, durch ein deutsches Kapitalkonsortium aufzukaufen und entsprechend stärker auf deutsche Interessenslagen hin zu positionie-

22 Vgl. dazu Kucher: Schicksal und Trauma, S. 45f. sowie ausführlicher: Leidinger et.al.: Habsburgs schmutziger Krieg.

23 Vgl. Hofmannsthal: Aufzeichnungen, S. 983f.

24 Aus der Rede Solfs anlässlich der Gründung der Deutschen Gesellschaft; zit. nach Lunzer: Hofmannsthals politische Tätigkeit, S. 170.

25 Hofmannsthal: Unsere Nationen, S. 274. In diesem Zusammenhang ist auch auf Gespräche zwischen Bodenhausen und Hofmannsthal zu verweisen, die, folgt man den Aufzeichnungen Bodenhausens, auf Hofmannsthals Position zur (austro)polnischen Frage, die anfangs mit jener von Andrian übereinstimmte und im erwähnten Essay deutlich zum Ausdruck kam, offenbar insofern einwirkte, als er sich Bodenhausens deutschimperialer Option angeschlossen hätte, die jener maßgeblich mit ökonomischen Argumenten untermauert habe. Vgl. Hofmannsthal: Aufzeichnungen, S. 992f. 
ren. ${ }^{26}$ Andererseits bewirkten diese Berliner Wochen, dass sich Hofmannsthal innerhalb kürzester Zeit mit den bereits erwähnten Kriegszieltexten vertraut machte und selbst an eine >Klub $<$-Gründung in Wien nachdachte, die jedoch aufgrund der Priorität seines Bibliothek-Projekts nicht über das Stadium einer Notiz hinauskam.

Es ist nicht entscheidend und kann mangels präziserer Hinweise auch nicht genauer ausgeführt werden, was Hofmannsthal von Rohrbach, Scheler, Munin (= Karl Iro) und Winterstetten im Sommer-Herbst 1915 genau gelesen und wie er deren Texte gelesen hat. Es genügt hier, deutlich zu machen, in welche Umgebung er sich hiermit begab und wie bzw. ob einzelne spätere Eintragungen, etwa die im Eingangszitat des vorliegenden Beitrags angegebene vom 28. Oktober 1915 (Brüssel) über die künftige Rolle auf dem Balkan, ${ }^{27}$ dazu in Beziehung gebracht werden können.

Zum ersten dieser Aspekte und Referenztexte: Paul Rohrbach, Publizist, Theologe und Kolonialbeamter, war Mitbegründer der 1914 eingerichteten Wochenschrift Das Größere Deutschland, die gemäß Untertitel explizit Deutsche Welt-und Kolonialpolitik thematisierte. Über seine zahlreichen Essays, die vor ihrer Buchpublikation ebendort veröffentlicht wurden, gewann Rohrbach bedeutenden Einfluss auf die Kriegszielpolitik der militärischpolitischen Eliten. ${ }^{28}$ Er stand auch - neben den wissenschafts- und kunstgeschichtlich prominenteren Figuren Hans Delbrück, Friedrich Meinecke und Ernst Jäckh - auf der Berlin-Besucherliste Hofmannsthals im Oktober 1915..$^{29}$ Nachgewiesen ist die Lektüre seiner Flugschrift Russland und wir (1914), die weitgehend unverändert in die Schrift Der Krieg und die deutsche Politik (1914) aufgenommen wurde. Hinsichtlich des Balkans werden dort im Abschnitt »Die russische Gefahr « die in zeitgenössischer Sicht bekannten und die Diskussion dominierenden Positionen, d.h. insbesondere die (freilich ungeprüft dargelegten) russischen außenpolitischen Absichten wie das Bestreben nach Schwächung des Osmanischen Reiches einerseits und der Aufbau einer Balkan-Föderation gegen Österreich-Ungarn andererseits bekräftigt. Zugleich würden diese Tendenzen die deutschen Expansionspläne Richtung Südosteuropa - Stichwort Berlin-Bagdad - gefährden und daher

Hofmannsthal: Aufzeichnungen, S. 986 mit Bezugnahme auf eine Tagebucheintragung von Josef Redlich, die sich auf ein Gespräch mit dem Wiener Korrespondenten der »Frankfurter Zeitung«, Hugo Ganz, beruft.

27 Hofmannsthal: Aufzeichnungen, S. 655.

28 U.a.publizierte dort auch der hochrangige Exponent des Alldeutschen Verbandes und Flottenoffizier Ernst Christian Graf zu Reventlow, der in der Weimarer Republik ein herausragender Vertreter der antisemitischen und revanchistisch orientierten Deutschvölkischen Freiheitspartei war, bevor er 1927 zur NSDAP wechselte. 
ein gemeinsames, koordiniertes Engagement erfordern. Ein Engagement, das im Rahmen der Maxime `Konzentration und Ausdehnung die einzige kontinentaleuropäische koloniale Option Deutschlands darstelle ${ }^{30}$ und somit - ohne es offen auszusprechen - gegen die defensiven $>$ Überleben in Würde`-Optionen Österreichs, denen situationselastische, d.h. temporär auch expansive Pläne Serbiens, Montenegros und Rumäniens gegenüber stehen, hierarchisiert wird. ${ }^{31}$

Berlin-Bagdad ist auch der Titel einer sehr erfolgreichen Schrift, die der aus Vorarlberg gebürtige und zunächst erfolglose Journalist, Jurist, gescheiterte Fabrikant, Schönerer-Schüler und Sekretär des Alldeutschen Verbands (1912-1914) Albert Ritter unter dem Pseudonym Karl von Winterstetten 1913 veröffentlicht und die zu Kriegsbeginn 1914 gerade die neunte Auflage erzielt hatte. Es handelt sich hierbei um eine immer wieder auf Rohrbach, aber auch offen auf Georg von Schönerer zurückgreifende Propagandaschrift mit z.T. krausen Verschwörungstheorien. Sie zielte letztlich darauf ab, Deutschland und Österreich-Ungarn zu einem »Schutz- und Trutzbündnis dauernd miteinander verbinden ", in dem Österreich unmissverständlich Souveränitätsrechte abgäbe, dafür aber der »Gefahr der Zertrümmerung durch die vereinten Kräfte des Slawentums « entgehen könne. ${ }^{32}$ Vor allem, und darin lag wohl die eigentliche Stoßrichtung, würde diese Konstellation dem Deutschen Reich die nötige politische wie wirtschaftliche Dimension verleihen und die ihm sonst durch England, so die Argumentation, versperrte Weltgeltung - den Weg nach Asien über den Balkan - in Aussicht stellen. Winterstetten übernimmt dabei unverhohlen Positionen aus der Schrift Deutscher Imperialismus (1912) von Arthur Dix, einem rechtskonservativen Ökonomen und Politiker, der, obgleich aus der Schule von Friedrich List kommend, 1914 das Konzept vom >Mitteleuropäischen StaatenbundImperialismus a ausarbeitete, ${ }^{33}$ wenn er davon spricht, es sei >unsere (d.h. der Deutschen) Sache, eine Umgestaltung Österreich-Ungarns in die Hände zu nehmen, die sich folgendermaßen anhört:

Bauern-Neuland, ein großes Wirtschaftsgebiet, Rettung des Deutschtums in der Donaumonarchie selbst, Einigung des Gesamtdeutschtums, offene Türe im Südosten und freien Weg für das Deutschtum auf seinen alten Pfaden, Schutz den nichtslawischen Südostvölkern vor dem Panslawismus - kurzum Berlin-Bagdad.

31 Dazu: Hekele: Kriegszielpolitik, S. 107.

32 Winterstetten: Berlin-Bagdad, S. 38.

33 A. Dix, 1875-1935, Verfasser der Schrift Raum und Rasse (1934) und Förderer von Hjalmar Schacht! 
Im Nachsatz wird schließlich unmissverständlich klar gestellt: »An der Durchführung der Aufgabe Berlin-Bagdad hängt das Schicksal unserer Rasse ${ }^{34}{ }^{34}$

$\mathrm{Zu}$ Max Scheler finden sich keine weiteren Eintragungen als der bloße Lektürevermerk. Es kann davon ausgegangen werden, dass Hofmannsthal in Schelers ausgreifendem Versuch, die Wurzeln des Krieges »in die Tiefen des organischen Lebens « zurückzuverfolgen und ihm eine geistig-moralische Begründung zu unterlegen, zumindest im Eingangsteil eine intellektuelle Wahlverwandtschaft erkennen konnte: »Aus dem Geiste entspringt und für den Geist entspringt der Krieg in seinem tiefsten Kern! - komme es ihm ja nicht primär auf »bloße physische Gewaltäußerung« an, sondern manifestiere sich in ihm eine legitime »Macht- und Willensauseinandersetzung der geistigen Kollektivpersönlichkeiten, die wir Staaten nennen... «. ${ }^{35}$ Eine Wahlverwandtschaft, die im Hinblick auf die Frage nach einem gerechten bzw. ungerechten Krieg (so der abschließende erste Teil bei Scheler) bereits auf Skepsis gestoßen sein dürfte, figurieren bei Scheler hierfür nämlich abstrakte Kategorien wie die »Größe und Kriegsgewichtigkeit der Gegensätze, die ihn treiben und die er ordnen soll ${ }^{36}{ }^{36}$ welche die Frage nach der machtpolitischen Hegemonie in Europa aufwerfen. Dieser Krieg sei gerecht, weil er "ein durch und durch politischer Krieg ist [...] ein Krieg um die Macht im Herzen der Welt, - ja um das Herz des Herzens der Welt, um die Hegemonie in Europa. $\ll^{37}$

Konzediert Scheler diesen Aspekt der Kriegsgewichtigkeit dem österreichisch-serbischen sowie dem österreichisch-deutsch-russischen Krieg, so stellt er sie für den deutsch-französischen immerhin in Frage, wäre dieser nämlich nur ein »in die alte romanisch-germanische Rassenfremdheit [!] eingebettete [...] Revanchekrieg « um verlorenes Terrain von 1870 und somit »ein absolutes sittliches Nonsens «. ${ }^{38}$ Andererseits müsse der Krieg - so er gerecht zu bezeichnen wäre (was im Fall Deutschlands fast immer in irgendeiner Weise zutreffe; Anm. d. Verf.) - »den echten Gemeinwillen (der volontè generale nicht der volontè de tous) der beteiligten Völker und Nationen entsprechen $« .{ }^{39}$

Spätestens der ins Konkretere zielende zweite Teil der Schrift dürfte bei Hofmannsthal wenig Begeisterung ausgelöst haben. Darin werden nämlich 
die von Russland und Serbien `verführten « slawischen Völker wiederholt als »Slavenhorden $\aleph^{40}$ denunziert, >Horden`, die immerhin einen beträchtlichen Anteil des österreichisch-ungarischen Staates verkörperten. Zugleich treten ihm hier klar die expansiven deutschen Interessen am Balkan als Schlüssel zum Orient und damit zur Weltpolitik zum Vorschein, wenn eine »Einheit des österreichisch-deutschen Wirtschaftssystems « unter der Maxime »freies Feld in den Orient $\aleph^{41}$ als lebensnotwendiger Riegel gegen den russischen Drang nach Konstantinopel, dem Mittelmeer und darüber hinaus gefordert, und der Idee eigenständiger >südösterreichischer`Interessen eine Absage erteilt wird. Spätestens hier wird sich Hofmannsthal an Gespräche mit Bodenhausen erinnert und seine Vorbehalte gegen eine drohende Kolonialisierung durch Deutschland bestätigt gefunden haben, die freilich in Wien selbst im Dezember 1915 durch ein von Hochschullehrern unterfertigtes Memorandum Stützung erhielten. ${ }^{42}$

Auch die Differenz zu Kriegszielschriften aus dem Freundeskreis, etwa den im August 1915 in der »Österreichischen Rundschau« erschienenen Überlegungen von Carl Brockhausen wird ihm hier vor Augen getreten sein, hieß es bei diesem doch immerhin:

Unser Kriegsziel ist nicht der Kampf um Weltherrschaft mit seinem unvermeidlichen Dauerkrieg, sondern Völkergemeinschaft, also Symbiose, als Voraussetzung des Dauerfriedens. Das Nebeneinanderleben Gleichberechtigter bedeutet den Sieg des österreichischen Staatsgedankens. ${ }^{43}$

4.

Ob die Enttäuschung, bei seinen deutschen Freunden wenig Verständnis zu finden für seine `österreichische Idee` letztlich dafür ausschlaggebend gewesen war, dass Hofmannsthal im Jahr 1916 in seinen Aufzeichnungen nur mehr einmal direkt auf den Krieg zu sprechen kam - und zwar anlässlich eines Lichtbildvortrages in Berlin zu den ersten Isonzo-Schlachten sowie eines Vortrags unter dem Titel Unser Krieg (vor großem Publikum, d. h. dem nahezu vollzählig erschienenen k.u.k. Botschafts- und Militärpersonal), in dem er, so der Bericht der »Neuen Freien Presse « und der » Vossischen Zeitung «, den deutschen Bundesgenossen eindringlich die »aus innerer

41 Ebd., S. 172.

42 Vgl. Rauchensteiner: Der Erste Weltkrieg, S. 501.

43 Zit. nach: Hofmannsthal: Reden und Aufsätze 3, S. $782 \mathrm{f}$. 
Kraft emporwachsende Improvisation« als Schlüssel des Überlebens dargelegt habe - ist zwar nicht gesichert, darf jedoch als These in den Raum gestellt werden. ${ }^{44}$ Damit hat Hofmannsthal an eine konzeptuelle Maxime angeknüpft, die sich wie ein roter Faden durch einige seiner Essays seit Geist der Karpathen (1915) zog. Sie sollte auch in dem auf Betreiben Andrians nach einigen bürokratischen Schwierigkeiten im Juli 1916 eingefädelten Warschauer Vortrag in einem »für Österreich mehr als peinlichen Moment « über »ein patriotisches Thema « eine Rolle spielen..$^{45}$ Von dieser Rede, hervorgegangen und improvisiert weiterentwickelt aus dem im März 1916 im Hause Nostiz gehaltenen Privatvortrag unter dem Titel Das Phänomen Österreich ${ }^{46}$ ist zwar keine verlässliche Abschrift erhalten geblieben, doch sie ist in sein essayistisches Werk eingegangen, nachbearbeitet unter dem unverfänglichen Titel Österreich im Spiegel seiner Dichtung. Die im Hinblick auf die prekäre militärische Lage an der Ostfront von Andrian so herbeigewünschte patriotische Ermunterung, nicht zuletzt aufgrund des hierzu eingeladenen deutschen Offizierskorps nebst ausgesuchten Vertretern der polnischen politisch-kulturellen Elite, hat Hofmannsthal dabei in einem eher unerwarteten, wenngleich, folgt man den Zeitungsberichten, erfolgreichen Ausweichmanöver über die Differenzen zwischen deutscher und österreichischer literarischer Kultur eingelöst. ${ }^{47}$ Neben der Wichtigkeit der Musik strich Hofmannsthal in seinem einleitenden Teil über Grillparzer gerade die Bedeutung seiner slawischen Amme heraus, »auf deren Schoß Grillparzer in dem Textbuche zur `Zauberflöte sein nachfolgendes, im »Hauch der Sagen von Drahomira, von Herzog Krok und seinen Töchtern « stehendes Werk. ${ }^{48}$ Er insistierte über die Bindung an die landschaftliche Dimension somit auf Volksnähe und Partikularismus oder, in moderner Diktion, auf kultureller Heterogenität und Polyphonie als zentralen Ressourcen. Diese würden sich nicht nur als ein bezaubernder "poetischer und reizvoller Weltzustand « in der Dichtung, sondern auch hier wohl, um seinem Publikum entgegenzukommen - in mitunter unerwarteten militärischen Tugenden äußern. Das auf sprachlicher Homogenität fußende >nationale Pathos` wolle er daher zugunsten der »Spontaneität»

Zit. nach der »Neuen Freien Presse« von 4.2.1916, S. 12; dazu: Hofmannsthal: Aufzeichnungen, S. 1017.

45 Hofmannsthal an Bahr, 17.6.1916, zit. nach Hofmannsthal: Reden und Aufsätze 3, S. 841.

46 Ebd., S. 811.

47 Vgl. dazu die Berichte im »Kurjer Polski« sowie in der »Deutschen Warschauer Zeitung« vom 9.7.1916; zit. ebd., S. $843 \mathrm{f}$.

48 Hofmannsthal: Österreich im Spiegel seiner Dichtung, S. 183. 
heterogener Individuen bei Seite lassen, denn: »Der Begriff der Nation darf nicht überanstrengt werden. « $^{49}$

Damit positionierte sich Hofmannsthal noch vor der Lektüre der einflussreichen Mitteleuropa-Schrift von Friedrich Naumann, die ihm Josef Redlich Ende November 1915 übergeben hatte und den er selbst im Jänner 1916 in Berlin treffen sollte, gegen dieses - von seinen deutschen Gesprächspartnern weitgehend mitgetragene - liberalnationale, de facto aber deutschhegemoniale Konzept eines um südosteuropäische Territorien erweiterten Staatenbundes, den Naumann vom ersten Kapitel an als »Zusammenschluß des Deutschen Reiches mit der österreichisch-ungarischen Doppelmonarchie « gedacht hat. ${ }^{50}$ Bei allem Verständnis für die komplizierte nationale und konfessionelle Verfasstheit der Doppelmonarchie werden hinsichtlich des angedacht Zusammenzuschließenden klare Prioritäten formuliert, und zwar dergestalt, dass letztlich »um das Deutschtum herum [...] die Kultur von Mitteleuropa [wächst] «, und dabei [soll] die »Natur der Völker [...] von uns erhöht werden durch ordnende Vernunft « - eine Maxime, die an anderen Stellen freilich auch durch jene der "Disziplin" ergänzt, wenn nicht substituiert wird. ${ }^{51}$

Inwieweit Hofmannsthal neben seinen eigenen Vorstellungen auch durch die im Frühjahr 1916 erfolgte Lektüre von Friedrich Meineckes Schrift Weltbürgertum und Nationalstaat, die 1915 neu aufgelegt worden war, in seinen verdeckt antinationalen Spitzen bestärkt worden war, lässt sich nicht mit Sicherheit ausmachen; die zeitliche Koinzidenz ist jedenfalls gegeben, obgleich in durchaus zentralen Texten Hofmannsthal ambivalente Argumentationen im Hinblick auf eine polykulturelle, übernationale wie auch deutschkulturelle Zugehörigkeit und Perspektive zugleich auszuloten scheint. $^{52}$

Faktum ist allerdings auch, dass Hofmannsthal 1916 den Großteil seiner Einträge der Lektüre französischer Klassiker seit Molière und der Wiederaufnahme von Lustspielplänen nebst einer großen ShakespeareHommage gewidmet hat - eine fast paradox anmutende Reaktion auf einen resonanzreichen Vortrag, der vermutlich die Erwartungen seines Publikums nicht wirklich erfüllt hat. Eine Antwort auf die im Zitat-Motto angezogene Frage, ob Österreich den Balkan beherrschen oder bloß ein belastendes Hinterland darstellen werde, hat sich Hofmannsthal selbst entweder nicht

50 Vgl. Hofmannsthal: Bibliothek, S. 504; Naumann: Mitteleuropa, S. 1, dazu auch Streim: Deutscher Geist und europäische Kultur, S. 174ff.

51 Naumann: Mitteleuropa, S. 62.

52 Hofmannsthal: Aufzeichnungen, S. $993 f$. 
geben können oder nicht geben wollen. Auffällig ist freilich, dass es gerade zum Krieg in Serbien, zur demütigenden Niederlage vom Dezember 1914 wie zur erfolgreichen Offensive vom Oktober 1915 (letztere allerdings nur mit deutscher Unterstützung ermöglicht), und damit zum Schlüsselraum jeglicher künftiger Balkanpolitik, keine Eintragungen oder essayistische Überlegungen Hofmannsthals gibt - auch nicht im Umfeld seines Vorworts zum Prinz Eugen-Buch von Paul Zifferer. ${ }^{53}$ Es empfiehlt sich daher, mit einer symptomatischen Eintragung vom Spätherbst 1917 zu schließen, in der Hofmannsthal im Kontext von Überlegungen zu seiner Gegenwart einerseits anmerkt: »deutschnationaler Sinn abstoßend «, andererseits »ein Anstarren der Vergangenheit « beklagt und das Nicht-Politische als den »beste[n] politische[n] Geist dieses Landes« auszumachen meint, um folgende Quintessenz daraus zu ziehen: »Rhythmus dieses Landes ist schwerer zu verstehen als jedes andren. ${ }^{54}$

Damit erledigt sich auch die Ausgangsfrage, insbesondere ihre zweite, auf eine mögliche dominante Rolle Österreichs am Balkan und im östlichen Mittelmeer abzielende Überlegung aus dem Jahr 1915 von selbst: Hofmannsthal konnte seit Mitte 1917 nach einem weiteren Berlinbesuch sowie einer Pragreise, die ihm in Gesprächen mit Bedřich Stepánek und Jaroslav Kvapil die irritierend desintegrative Kraft der entstehenden tschechischen Nationsvorstellungen vor Augen führte, ${ }^{55}$ keine Sinnhaftigkeit mehr in jeglicher Kriegszielpolitik erkennen. Ihr hielt er wohl noch in Vorträgen mit Verve und Verzweiflung die Idee Europa als kulturpolitisches, durch die Existenz Österreichs mitverbürgtes >Mission Statement k künftigen Zusammenlebens entgegen, eine Idee, die er auch umgehend ästhetisierte: $[\ldots]$ muß einem immer wieder das Schöne hervorsteigen, aus dieser Hölle um uns «. ${ }^{56}$ Angesichts der Wahrnehmung einer tiefen "geistige[n] Zerrissenheit « ${ }^{57}$ sowie des sich abzeichnenden dramatischen Bruchs mit den slawischen Nationen seit seiner Pragerfahrung schmolz ihm freilich die Aussicht auf Verwirklichung jenes Schönen von Tag zu Tag, weshalb Ende 1917 unter dem Stichwort `Zukunft einerseits von »Enttäuschungen des Lebens« die Rede ist, andererseits nur mehr, und dies auch 1918, Lektüren, Annotationen und flüchtige Begegnungen aufgelistet erscheinen.

Vgl. Hofmannsthal: Reden und Aufsätze 3, S. 798. Auch der am 3.12.1915 in der »Neuen Freien Presse « veröffentlichte Vorabdruck nahm auf die noch frischen Eindrücke über den militärischen Erfolg in Serbien keinen ersichtlichen Bezug.

54 Ebd., S. $749 f$.

55 Vgl. Lunzer: Hofmannsthals politische Tätigkeit, S. 241f.

56 Hofmannsthal/Bodenhausen: Briefe der Freundschaft, Brief vom 10.7.1917, S. 236.

57 Vgl. Hofmannsthal: In Erwartung der Zukunft. In: ders.: Erfundene Gespräche und Briefe, S. 196. 


\section{Literaturverzeichnis}

Andrian, Leopold von: Korrespondenzen, Notizen, Essays, Berichte. Hgg. Ursula Prutsch, Klaus Zeyringer. Wien, Köln, Weimar: Böhlau 2003.

Anonym: Die Eröffnung der Siegel. »Neue Freie Presse« (1.11.1915). <http://anno.onb. ac.at $/$ cgi-content $/$ anno? aid $=$ nfp\&datum $=19150101 \&$ seite $=1 \&$ zoom $=33>($ Zugriff 3.11.2015).

Hekele, Martin: Die Kriegszielpolitik der österreichisch-ungarischen Monarchie im Ersten Weltkrieg. Wien: Diss. Phil. maschinschriftlich 1996.

Hofmannsthal, Hugo v.: Appell an die höheren Stände. In: Sämtliche Werke. Kritische Ausgabe. Bd. XXXIV. Reden und Aufsätze 3, 1910-1919. Hgg. Klaus E. Bohnenkamp, Katja Kaluga, Klaus-Dieter Krabiel. Frankfurt/M.: S. Fischer 2011.

Hofmannsthal, Hugo v.: Aufbauen, nicht einreißen. »Neue Freie Presse« (1.11.1915). <http:// anno.onb.ac.at $/$ cgi-content $/$ anno aid $=n f p \&$ datum $=19150101 \&$ seite $=3 \& z o o m=33>$ (Zugriff 3.11.2015).

Hofmannsthal, Hugo v.: In Erwartung der Zukunft. In: ders.: Sämtliche Werke. Kritische Ausgabe. Bd. XXXI. Erfundene Gespräche und Briefe. Hg. Ellen Ritter. Frankfurt/M.: S. Fischer 1991.

Hofmannsthal, Hugo v.: Österreich im Spiegel seiner Dichtung. ders.: Sämtliche Werke. Kritische Ausgabe. Bd. XXXIV. Reden und Aufsätze 3, 1910-1919. Hgg. Klaus E. Bohnenkamp, Katja Kaluga, Klaus-Dieter Krabiel. Frankfurt/M.: S. Fischer 2011.

Hofmannsthal, Hugo v.: Sämtliche Werke. Kritische Ausgabe. Bd. XXXI. Erfundene Gespräche und Briefe. Hg. Ellen Ritter. Frankfurt/M.: S. Fischer 1991.

Hofmannsthal, Hugo v.: Sämtliche Werke. Kritische Ausgabe. Bd. XXXVIII. Aufzeichnungen. Hgg. Rudolf Hirsch, Ellen Ritter, in Zusammenarbeit mit Konrad Heumann und Peter Michael Braunwarth. Frankfurt/M.: S. Fischer 2013.

Hofmannsthal, Hugo v.: Sämtliche Werke. Kritische Ausgabe. Bd. XL. Bibliothek. Hg. Ellen Ritter in Zusammenarbeit mit Dalia Bukauskaite und Konrad Heumann. Frankfurt/M.: S. Fischer 2011.

Hofmannsthal, Hugo v.: Unsere Militärverwaltung in Polen. In: ders.: Sämtliche Werke. Kritische Ausgabe. Bd. XXXIV. Reden und Aufsätze 3, 1910-1919. Hgg. Klaus E. Bohnenkamp, Katja Kaluga, Klaus-Dieter Krabiel. Frankfurt/M.: S. Fischer 2011.

Hofmannsthal, Hugo v.: Unsere Nationen. In: ders.: Sämtliche Werke. Kritische Ausgabe. Bd. XXXIV. Reden und Aufsätze 3, 1910-1919. Hgg. Klaus E. Bohnenkamp, Katja Kaluga, Klaus-Dieter Krabiel. Frankfurt/M.: S. Fischer 2011.

Hofmannsthal, Hugo v., Bodenhausen Eberhard: Briefe der Freundschaft. Jena: Eugen Diederichs 1953.

Hofmannsthal, Hugo v., Gräfin Degenfeld, Ottonie: Briefwechsel. Hg. Marie Therese MillerDegenfeld. Frankfurt/M.: Fischer 1974.

Hofmannsthal, Hugo v., Graf Kessler, Harry. Briefwechsel 1898-1929. Hg. Hilde Burger, Frankfurt/M.: Insel 1968.

Hofmannsthal, Hugo v., Redlich Josef: Briefwechsel. Hg. Helga Ebner-Fußgänger. Frankfurt/M.: Fischer 1971.

Hofmannsthal, Hugo von, Schnitzler, Arthur: Briefwechsel. Hgg. Therese Nickel, Heinrich Schnitzler. Frankfurt/M.: Fischer 1964. 2. Aufl. 1983.

Kraus, Karl: Gruß an Bahr und Hofmannsthal. »Die Fackel« Nr. 423-425, 5.5.1916, S. 41-52. Kraus, Karl: Weltgericht. Leipzig: Kurt Wolff 1919. 
Kucher, Primus-Heinz: Schicksal und Trauma. Galizien und die Karpathen in der Wahrnehmung österreichischer Autoren zu Beginn des Ersten Weltkrieges. »informationen zur deutschdidaktik. Zeitschrift für den Deutschunterricht in Wissenschaft und Schule« 2 (2015): Kulturen des Erinnerns. Innsbruck: Studien Verlag 2015, S. 38-48.

Leidinger, Hannes; Moritz, Verena; Moser, Karin; Dominik, Wolfram (Hgg.): Habsburgs schmutziger Krieg. Ermittlungen zur österreichisch-ungarischen Kriegsführung 19141918. St. Pölten: Residenzverlag 2014.

Lunzer, Heinz: Hofmannsthals politische Tätigkeit in den Jahren 1914-1917. Bern, Frankfurt/M.: P. Lang 1981.

Naumann, Friedrich: Mitteleuropa. Berlin: Georg Reimer 1915.

Nordau, Max: Silversternacht. »Neue Freie Presse« (1.11.1915). <http://anno.onb.ac.at/cgicontent/anno? aid $=$ nfp\&datum $=19150101 \&$ seite $=3 \&$ zoom $=33>($ Zugriff 3.11 .2015$)$.

Propst, Maximilian: Harry Graf Kessler. »Die Zeit« 8 (13.2.2014). Online-Version: <http:// www.zeit.de/2014/08/erster-weltkrieg-harry-graf-kessler> (Zugriff 3.11.2015).

Rauchensteiner, Manfried: Der Erste Weltkrieg und das Ende der Habsburgermonarchie. Wien, Köln, Weimar: Böhlau 2013.

Rohrbach, Paul: Der Krieg und die deutsche Politik. Dresden: Das Größere Deutschland 1914.

Scheichl, Sigurd Paul: Karl Kraus' Weltgericht - Eine Bilanz. »Jahrbuch/Yearbook Simon Dubnow Institut « XIII. Göttingen: V\&R 2014, S. 285-308.

Scheler, Max: Der Genius des Krieges und der Deutsche Krieg. Leipzig: Verlag der Weißen Bücher 1915.

Streim, Georg: Deutscher Geist und europäische Kultur. Die seuropäische Idee in der Kriegszielpolitik von Rudolf Borchardt, Hugo von Hofmannsthal und Rudolf Pannwitz. »Germanisch-romanische Monatsschrift« N.F. 46 (1996), S. 174-197.

Winterstetten, Karl: Berlin-Bagdad. Neue Ziele mitteleuropäischer Politik. 9. Aufl. München: Lehmann 1914. 